\title{
3D structure of hadrons by generalized distribution amplitudes and gravitational form factors
}

\author{
S. Kumano ${ }^{* a, b}$, Qin-Tao Song ${ }^{a}$, and O. V. Teryaev ${ }^{c}$ \\ ${ }^{a}$ KEK Theory Center, Institute of Particle and Nuclear Studies, KEK, \\ and Department of Particle and Nuclear Physics, Graduate University for Advanced Studies \\ (SOKENDAI), Ooho 1-1, Tsukuba, Ibaraki, 305-0801, Japan \\ ${ }^{b}$ J-PARC Branch, KEK Theory Center, Institute of Particle and Nuclear Studies, KEK, \\ and Theory Group, Particle and Nuclear Physics Division, J-PARC Center, \\ 203-1, Shirakata, Tokai, Ibaraki, 319-1106, Japan \\ ${ }^{c}$ Bogoliubov Laboratory of Theoretical Physics, Joint Institute for Nuclear Research, \\ 141980 Dubna, Russia
}

Generalized distribution amplitudes (GDAs) are one type of three-dimensional structure functions, and they are related to the generalized distribution functions (GPDs) by the $s-t$ crossing of the Mandelstam variables. The GDA studies provide information on three-dimensional tomography of hadrons. The GDAs can be investigated by the two-photon process $\gamma^{*} \gamma \rightarrow h \bar{h}$, and the GPDs are studied by the deeply virtual Compton scattering $\gamma^{*} h \rightarrow \gamma h$. The GDA studies had been pure theoretical topics, although the GPDs have been experimentally investigated, because there was no available experimental measurement. Recently, the Belle collaboration reported their measurements on the $\gamma^{*} \gamma \rightarrow \pi^{0} \pi^{0}$ differential cross section, so that it became possible to find the GDAs from their measurements. Here, we report our analysis of the Belle data for determining the pion GDAs. From the GDAs, the timelike gravitational form factors $\Theta_{1}(s)$ and $\Theta_{2}(s)$ can be calculated, which are mechanical (pressure, shear force) and mass (energy) form factors, respectively. They are converted to the spacelike form factors by using the dispersion relation, and then gravitational radii are evaluated for the pion. The mass and mechanical radii are obtained from $\Theta_{2}$ and $\Theta_{1}$ as $\sqrt{\left\langle r^{2}\right\rangle_{\text {mass }}}=0.56 \sim 0.69 \mathrm{fm}$ and $\sqrt{\left\langle r^{2}\right\rangle_{\text {mech }}}=1.45 \sim 1.56 \mathrm{fm}$, whereas the experimental charge radius is $\sqrt{\left\langle r^{2}\right\rangle_{\text {charge }}}=0.672 \pm 0.008 \mathrm{fm}$ for the charged pion. Future developments are expected in this new field to explore gravitational physics in the quark and gluon level.

XXV International Workshop on Deep-Inelastic Scattering and Related Subjects 3-7 April 2017

University of Birmingham, $U K$

\footnotetext{
*Speaker.
} 


\section{Introduction}

Understanding of three-dimensional (3D) structure functions for the nucleon is one of hot topics in hadron physics. One of the major purposes of their studies is to understand the origin of nucleon spin including partonic orbital-angular-momentum contributions. However, they could be used for other purposes such as possible internal structure studies of exotic hadron candidates [四]. Now, theoretical and experimental studies are focused on the generalized distribution functions (GPDs) and transverse-momentum-dependent parton distributions (TMDs) as the 3D structure functions by deeply virtual Compton scattering and semi-inclusive lepton scattering processes. There is another type of 3D structure functions called generalized distribution amplitudes (GDAs) which can be measured by timelike processes, typically by the two-photon process $\gamma^{*} \gamma \rightarrow h \bar{h}$ to produce a hadron pair in the final state [వ]. It is the $s-t$ crossed channel to the virtual Compton scattering $\gamma^{*} h \rightarrow \gamma h$. There was no experimental measurement for studying the GDAs until recently, so that they had been investigated as a theoretical subject. However, time has come to find the GDAs for hadrons because the Belle collaboration reported measurements on the two-photon process $\gamma^{*} \gamma \rightarrow \pi^{0} \pi^{0}$ recently [B]]. It became possible to extract appropriate GDAs from experimental measurements. Once the GDAs are obtained, it is possible to calculate gravitational form factors for hadrons.

Here, we determine quark GDAs and gravitational form factors for $\pi^{0}$ from an analysis of the Belle data in the leading order of the QCD running coupling constant and the leading twist [ [ 4 ]. This is the first attempt to extract the GDAs and the gravitational form factors from actual measurements. In Sec. [, we explain our formalism to describe the $\gamma^{*} \gamma \rightarrow \pi^{0} \pi^{0}$ cross section in terms of the GDAs. In Sec. 1], our analysis results are shown for the GDAs, and then the gravitational form factors and radii are calculated from the determined GDAs. Our studies are summarized in Sec. 团.

\section{Theoretical formalism}

\subsection{GDAs and gravitational form factors}

The GPDs can be measured by the deeply virtual Compton scattering (DVCS) shown in Fig.1 (a), whereas the the GDAs can be investigated by the two-photon process in Fig.1(b). They are related by the $s-t$ crossing of the Mandelstam variables. The pion, photon, and quark momenta

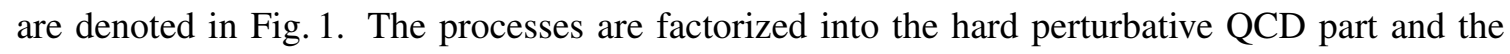

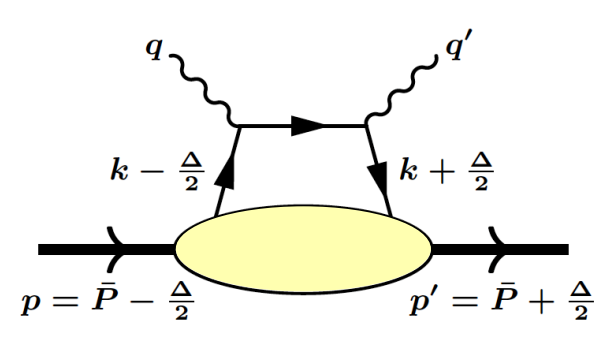

(a)

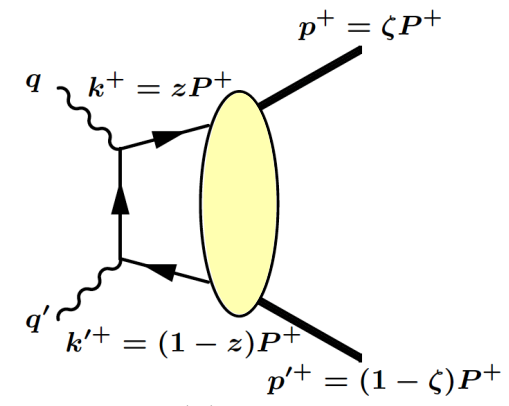

(b)

Figure 1: $(a)$ Virtual Compton process to probe GPDs $(b)$ Two-photon process to probe GDAs. 
soft one described by the GPDs and GDAs if the conditions, $Q^{2} \gg|t|, \Lambda_{\mathrm{QCD}}^{2}$ for the DVCS and $Q^{2} \gg W^{2}, \Lambda_{\mathrm{QCD}}^{2}$ for the two photon process, are satisfied. The variables $t$ and $W^{2}$ are defined by $t=\left(p-p^{\prime}\right)^{2}$ and $W^{2}=\left(p+p^{\prime}\right)^{2}=s$. The pion GPDs, for example for $\pi^{0}$, are defined by off-forward matrix elements of quark and gluon operators with a lightcone separation as

$$
\left.\int \frac{d y^{-}}{4 \pi} e^{i x \bar{P}^{+} y^{-}}\left\langle\pi^{0}\left(p^{\prime}\right)\left|\bar{q}(-y / 2) \gamma^{+} q(y / 2)\right| \pi^{0}(p)\right\rangle\right|_{y^{+}=\vec{y}_{\perp}=0}=H_{q}^{\pi}(x, \xi, t) .
$$

Here, $x$ is given by $x=Q^{2} /(2 p \cdot q)$ with $Q^{2}=-q^{2}$, and $\xi$ is the skewdness parameter given by $\xi=\bar{Q}^{2} /(2 \bar{P} \cdot \bar{q})$ with $\bar{q}=\left(q+q^{\prime}\right) / 2, \bar{P}=\left(p+p^{\prime}\right) / 2$, and $\bar{Q}^{2}=-\bar{q}^{2}$. The link operator for the color gauge invariance is not explicitly written in Eq. (ل्.] just for simplicity. The pion is not a stable target, so that the usual DVCS process cannot be used for measuring its GPDs. Nevertheless, we show the definition in order to compare with the pion GDAs, which are investigated in this work. The quark GDAs are defined by the matrix element of the same operator in defining the GPDs between the vacuum and the hadron pair:

$$
\Phi_{q}^{\pi^{0} \pi^{0}}\left(z, \zeta, W^{2}\right)=\left.\int \frac{d y^{-}}{2 \pi} e^{i(2 z-1) P^{+} y^{-} / 2}\left\langle\pi^{0}(p) \pi^{0}\left(p^{\prime}\right)\left|\bar{q}(-y / 2) \gamma^{+} q(y / 2)\right| 0\right\rangle\right|_{y^{+}=\vec{y}_{\perp}=0} .
$$

Here, the variable $z$ is defined by $z=k \cdot q^{\prime} /\left(P \cdot q^{\prime}\right)$ with the total momentum $P=p+p^{\prime}$, and $\zeta$ is given by $\zeta=p \cdot q^{\prime} /\left(P \cdot q^{\prime}\right)=(1+\beta \cos \theta) / 2$ with the pion velocity $\beta=|\vec{p}| / p^{0}=\sqrt{1-4 m_{\pi}^{2} / W^{2}}$ and the scattering angle $\theta$ in the center-of-mass frame of final pions.

The GPDs and GDAs contain information on form factors of not only electromagnetic interactions but also gravitational interactions. It is understood by taking $n$-th moments of the GPDs and GDAs. For example, they are given for the GDAs as

$\left.2\left(P^{+} / 2\right)^{n} \int_{0}^{1} d z(2 z-1)^{n-1} \int \frac{d y^{-}}{2 \pi} e^{i(2 z-1) P^{+} y^{-} / 2} \bar{q}(-y / 2) \gamma^{+} q(y / 2)\right|_{y^{+}=\vec{y}_{\perp}=0}=\bar{q}(0) \gamma^{+}\left(i \overleftrightarrow{\partial}^{+}\right)^{n-1} q(0)$

where the derivative $\overleftrightarrow{\partial}$ is defined by $f_{1} \overleftrightarrow{\partial} f_{2}=\left[f_{1}\left(\partial f_{2}\right)-\left(\partial f_{1}\right) f_{2}\right] / 2$. Equation (2.3) indicates that the operator is the vector-type electromagnetic current and the energy-momentum tensor of a quark for $n=1$ and $n=2$, respectively, as shown in Fig.】. Therefore, if the GPDs and/or GDAs are determined, the gravitational form factors are obtained from them.

In fact, the second moments of the $\pi^{0}$ GDAs are given by

$$
\int_{0}^{1} d z(2 z-1) \Phi_{q}^{\pi^{0} \pi^{0}}\left(z, \zeta, W^{2}\right)=\frac{2}{\left(P^{+}\right)^{2}}\left\langle\pi^{0}(p) \pi^{0}\left(p^{\prime}\right)\left|T_{q}^{++}(0)\right| 0\right\rangle
$$

where the quark energy-momentum tensor is $T_{q}^{\mu v}(x)=\bar{q}(x) \gamma^{\left(\mu_{i} \overleftrightarrow{D}^{v)}\right.} q(x)$. Here, $D^{\mu}$ is the covariant derivative $D^{\mu}=\partial^{\mu}-i g \lambda^{a} A^{a, \mu} / 2$ defined by the QCD coupling constant $g$ and the SU(3) 
Gell-Mann matrix $\lambda^{a}$. Using the momenta $P=p+p^{\prime}$ and $\Delta=p^{\prime}-p$, we express the matrix element of the energy momentum tensor in terms of the gravitational form factors $\Theta_{1}$ and $\Theta_{2}$ as

$$
\left\langle\pi^{0}(p) \pi^{0}\left(p^{\prime}\right)\left|T_{q}^{\mu v}(0)\right| 0\right\rangle=\frac{1}{2}\left[\left(s g^{\mu v}-P^{\mu} P^{v}\right) \Theta_{1, q}(s)+\Delta^{\mu} \Delta^{v} \Theta_{2, q}(s)\right] .
$$

Therefore, if the GDAs (or GPDs) are determined, we can obtain the gravitational form factors of the pion and subsequently its gravitational radii. It is interesting to investigate the gravitational radii of hadrons in comparison with charge radii because their physics origins are different.

\section{$2.2 \gamma^{*} \gamma \rightarrow \pi^{0} \pi^{0}$ cross section in terms of GDAs}

The cross section for the pion-pair production process $\gamma^{*}(q) \gamma\left(q^{\prime}\right) \rightarrow \pi^{0}(p) \pi^{0}\left(p^{\prime}\right)$ is expressed by the matrix element $\mathscr{M}$ as

$$
d \sigma=\frac{1}{4 q \cdot q^{\prime}} \sum_{\lambda, \lambda^{\prime}}\left|\mathscr{M}\left(\gamma^{*} \gamma \rightarrow \pi^{0} \pi^{0}\right)\right|^{2} \frac{d^{3} p}{(2 \pi)^{3} 2 E_{p}} \frac{d^{3} p^{\prime}}{(2 \pi)^{3} 2 E_{p^{\prime}}}(2 \pi)^{4} \delta^{4}\left(q+q^{\prime}-p-p^{\prime}\right) .
$$

Here, one of the initial photons is taken on mass shell $\left(q^{\prime 2}=0\right)$. The matrix element $\mathscr{M}\left(\gamma^{*} \gamma \rightarrow\right.$ $\left.\pi^{0} \pi^{0}\right)$ is given by the hadron tensor $\mathscr{T}_{\mu \nu}$ and the photon polarization vector $\varepsilon^{\mu}(\lambda)$ as $i \mathscr{M}\left(\gamma^{*} \gamma \rightarrow\right.$ $\left.\pi^{0} \pi^{0}\right)=\varepsilon^{\mu}(\lambda) \varepsilon^{\nu}\left(\lambda^{\prime}\right) \mathscr{T}_{\mu \nu}$, where $\mathscr{T}_{\mu \nu}$ is expressed by the electromagnetic current $J_{\mu}^{e m}(y)$, and then it is given by the quark GDAs as

$$
\begin{aligned}
\mathscr{T}_{\mu \nu} & =i \int d^{4} y e^{-i q \cdot y}\left\langle\pi^{0}(p) \pi^{0}\left(p^{\prime}\right)\left|T J_{\mu}^{e m}(y) J_{v}^{e m}(0)\right| 0\right\rangle \\
& =-g_{T}^{\mu \nu} e^{2} \sum_{q} \frac{e_{q}^{2}}{2} \int_{0}^{1} d z \frac{2 z-1}{z(1-z)} \Phi_{q}^{\pi^{0} \pi^{0}}\left(z, \zeta, W^{2}\right) .
\end{aligned}
$$

in the leading order and leading twist with $g_{T}^{\mu \nu}=-1$ for $\mu=v=1,2$ and $g_{T}^{\mu \nu}=0$ for $\mu, v=$ others. The quark GPDs are expressed as

$$
\Phi_{q}^{\pi^{0} \pi^{0}}\left(z, \zeta, W^{2}\right)=N_{\alpha} z^{\alpha}(1-z)^{\alpha}(2 z-1)\left[\widetilde{B}_{10}\left(W^{2}\right)+\widetilde{B}_{12}\left(W^{2}\right) P_{2}(\cos \theta)\right],
$$

where S- and D-wave terms are expressed as $\widetilde{B}_{10}\left(W^{2}\right)$ and $\widetilde{B}_{12}\left(W^{2}\right)$, and $P_{2}(\cos \theta)$ is the Legendre polynomial. The functions $\widetilde{B}_{n l}\left(W^{2}\right)$ are described by the continuum term and resonance ones: $\widetilde{B}_{10}\left(W^{2}\right)=$ continuum + resonance $\left(f_{0}\right), \widetilde{B}_{12}\left(W^{2}\right)=$ continuum + resonance $\left(f_{2}\right)$. We try to fix the resonance terms as much as possible from other theoretical and experimental studies. The GPDs are expressed by a number of parameters, which are determined by a $\chi^{2}$ analysis of the Belle experimental data for $\gamma^{*} \gamma \rightarrow \pi^{0} \pi^{0}$. Because of the page limitation, we do not explain the details of the GDA parametrization. One of the parameters is the cutoff $\Lambda$ in the overall form factor of the continuum part $F_{q}^{\pi}\left(W^{2}\right)=1 /\left[1+\left(W^{2}-4 m_{\pi}^{2}\right) / \Lambda^{2}\right]^{n-1}$ with the constituent-counting factor $n=2$ [可]. The details should be found in Ref. [䧃].

\section{Determination of GDAs and gravitational form factors for pion}

Our analysis results are compared with the Belle data on $\gamma^{*} \gamma \rightarrow \pi^{0} \pi^{0}$ in Fig. B. First, the $f_{0}(980)$ resonance is not included in our analysis. The decay constant of $f_{0}(980)$ has been calculated theoretically by assuming that it is a $q \bar{q}$ state. If such $q \bar{q}$-type decay constant is used, it is 
much larger than the Belle data at $W \simeq 1 \mathrm{GeV}$. It means that $f_{0}(980)$ should not be interpreted by the $q \bar{q}$ configuration and it is possibly a tetraquark hadron or a $K \bar{K}$ molecule as other studies indicate [焑]. In Fig. B, the dotted curves indicate our cross sections without $f_{0}(500)$, and the solid ones are obtained by including a $f_{0}(500)$ contribution. It is difficult to interpret the data without $f_{0}(500)$ at small $W(<0.8 \mathrm{GeV})$. The overall agreement is obtained with the experimental measurements.

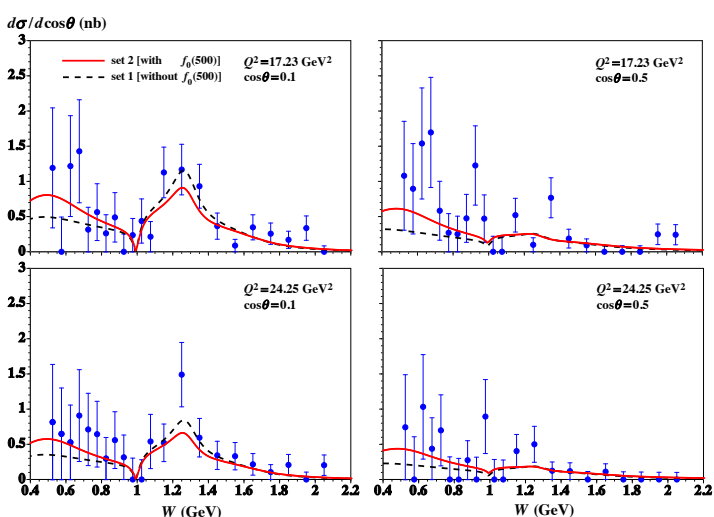

Figure 3: Comparison with Belle data [四].

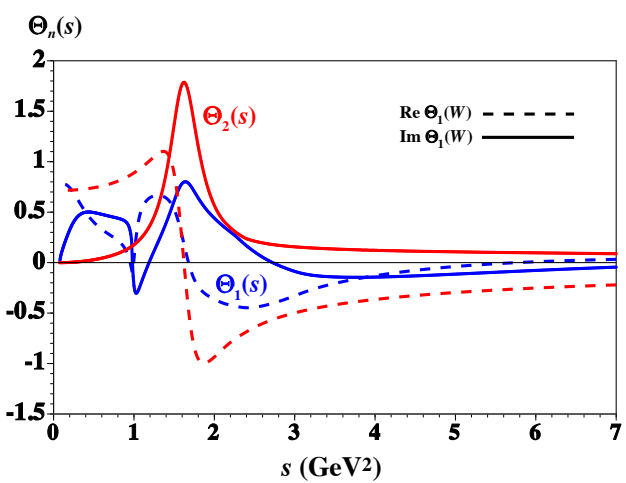

Figure 4: Timelike gravitational form factors for $\pi$ [䧃]

Once the GPDs are obtained, we can calculate the timelike gravitational form factors of the pion by using Eqs. (2.4) and ([2.5). Our results are shown in Fig. 因. Since it is a timelike process, the form factors have imaginary parts. The form factor $\Theta_{2}$ comes from the D-wave term, and it is peaked at the $f_{2}(1270)$ resonance. On the other hand, the S-wave term also contributes to $\Theta_{1}$ in addition to the D-wave one, so that the curves indicate complicated interference patterns. The timelike gravitational form factors are converted to the spacelike ones by using the dispersion relation, and the results are shown in Fig. $1(a)$. Taking the Fourier transforms of these functions, we obtain the space-coordinate densities $\rho_{1}(r)$ and $\rho_{2}(r)$ for $\Theta_{1}$ and $\Theta_{2}$, respectively, in Fig. $1(b)$.

In order to understand the physics meaning of the form factors and their densities, we may define the static energy-momentum tensor as $T_{q}^{\mu v}(\vec{r})=\int d^{3} q /\left[(2 \pi)^{3} 2 E\right] e^{i \vec{q} \cdot \vec{r}}\left\langle\pi^{0}\left(p^{\prime}\right)\left|T_{q}^{\mu v}(0)\right| \pi^{0}(p)\right\rangle$, where $E=\sqrt{m_{\pi}^{2}+\vec{q}^{2} / 4}\left[\right.$ []]. The $\mu v=00$ component satisfies the mass relation $\int d^{3} r T_{q}^{00}(\vec{r})=$ $m_{\pi} \Theta_{2, q}(0)$, so that $\Theta_{2}$ and $\rho_{2}(r)$ reflect the mass (energy) distributions in the pion. The $\mu \nu=i j$ $(i, j=1,2,3)$ components are expressed by the pressure $p(r)$ and shear force $s(r)$ as $T_{q}^{i j}(\vec{r})=$ $p_{q}(r) \delta_{i j}+s_{q}(r)\left(r_{i} r_{j} / r^{2}-\delta_{i j} / 3\right)$, which is expressed solely by $\Theta_{1}$. Therefore, $\Theta_{1}$ and $\rho_{1}(r)$ con-

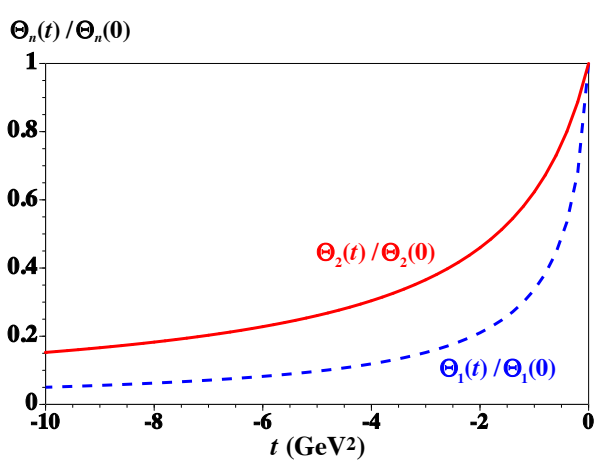

(a)

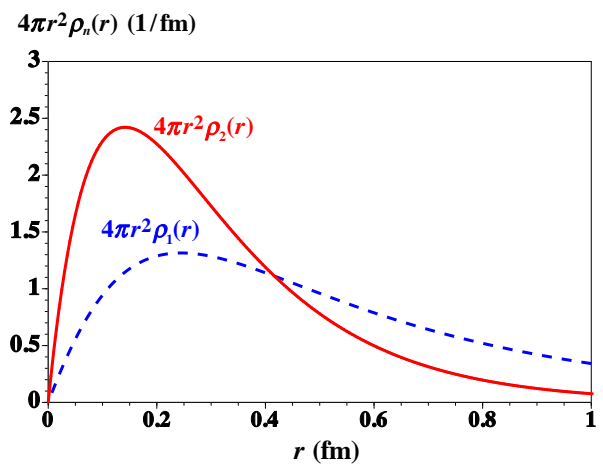

(b)

Figure 5: (a) Spacelike gravitational form factors (b) Mass and mechanical densities [四]. 
tain information on pressure and shear-force distributions in the pion.

From the densities, the gravitational mass and mechanical (pressure, shear force) radii are calculated from $\rho_{2}(r)$ (or $\Theta_{2}(t)$ ) and $\rho_{1}(r)$ (or $\Theta_{1}(t)$ ). We obtained

$$
\sqrt{\left\langle r^{2}\right\rangle_{\text {mass }}}=0.56 \sim 0.69 \mathrm{fm}, \quad \sqrt{\left\langle r^{2}\right\rangle_{\text {mech }}}=1.45 \sim 1.56 \mathrm{fm} .
$$

They indicate that the mass radius is similar or slightly smaller than the charge radius $\sqrt{\left\langle r^{2}\right\rangle_{\text {charge }}}=$ $0.672 \pm 0.008 \mathrm{fm}$ and that the mechanical radius is larger. This is the first finding on gravitational radii from actual experimental measurements. In comparison with the charge radius, much details should be investigated further.

We believe that this kind of field has a bright prospect in understanding gravitational physics in terms of quarks and gluons with experimental justifications. The KEK-B factory has just upgraded, and the errors of Fig. [ [ ] should be significantly reduced in the near future. Furthermore, other hadron-pair production processes are being analyzed by the Belle collaboration, and the future ILC project can probe different kinematical regions of the GDAs, especially the continuum part. The GDA studies had been purely theoretical projects for a long time; however, time has come to investigate them in comparison with experimental measurements and to explore gravitational physics in the quark and gluon level.

\section{Summary}

We have determined the pion GDAs from the KEKB measurements on $\gamma^{*} \gamma \rightarrow \pi^{0} \pi^{0}$. From the obtained GDAs, the timelike gravitational form factors $\Theta_{1}(s)$ and $\Theta_{2}(s)$ are obtained. Using the dispersion relation, we calculated spacelike gravitational form factors $\Theta_{1}(t)$ and $\Theta_{2}(t)$ and spacial densities $\rho_{1}(r)$ and $\rho_{2}(r)$. The functions $\Theta_{2}(t)\left[\rho_{2}(r)\right]$ and $\Theta_{1}(t)\left[\rho_{1}(r)\right]$ indicate gravitational mass (energy) and mechanical (pressure, shear force) distributions, respectively. From these distributions, the gravitational radii were determined for the pion. They are $\sqrt{\left\langle r^{2}\right\rangle_{\text {mass }}}=0.56 \sim 0.69 \mathrm{fm}$ and $\sqrt{\left\langle r^{2}\right\rangle_{\text {mech }}}=1.45 \sim 1.56 \mathrm{fm}$. The mass radius is similar to the charge radius $0.672 \pm 0.008 \mathrm{fm}$ or slightly smaller, and the mechanical radius is larger. This kind of studies have bright prospect in creating the field of gravitational physics in the elementary quark and gluon level.

\section{Acknowledgement}

This work was supported by Japan Society for the Promotion of Science (JSPS) Grants-in-Aid for Scientific Research (KAKENHI) Grant Number JP25105010.

\section{References}

[1] H. Kawamura and S. Kumano, Phys. Rev. D 89, 054007 (2014).

[2] M. Diehl, T. Gousset, B. Pire, and O. Teryaev, Phys. Rev. Lett. 81, 1782 (1998); For a review, see M. Diehl, Phys. Rept. 388, 41 (2003).

[3] M. Masuda et al. (Belle Collaboration), Phys. Rev. D 93, 032003 (2016).

[4] S. Kumano, Qin-Tao Song, and O. V. Teryaev, arXiv:1711.08088 [hep-ph].

[5] H. Kawamura, S. Kumano, and T. Sekihara, Phys. Rev. D 88, 034010 (2013). W.-C. Chang, S. Kumano, and T. Sekihara, Phys. Rev. D 93, 034006 (2016).

[6] S. Kumano, V. R. Pandharipande, Phys. Rev. D 38, 146 (1988); F. E. Close, N. Isgur, and S. Kumano, Nucl. Phys. B 389, 513 (1993); T. Sekihara and S. Kumano, Phys. Rev. D 92, 034010 (2015).

[7] M. V. Polyakov, Phys. Lett. B 555, 57 (2003). 